\title{
Global Well-Posedness and Long Time Decay of Fractional Navier-Stokes Equations in Fourier-Besov Spaces
}

\author{
Weiliang Xiao, ${ }^{1}$ Jiecheng Chen, ${ }^{2}$ Dashan Fan, ${ }^{3}$ and Xuhuan Zhou ${ }^{1}$ \\ ${ }^{1}$ Department of Mathematics, Zhejiang University, Hangzhou 310027, China \\ ${ }^{2}$ Department of Mathematics, Zhejiang Normal University, Jinhua 321000, China \\ ${ }^{3}$ Department of Mathematics, University of Wisconsin-Milwaukee, Milwaukee, WI 53201, USA
}

Correspondence should be addressed to Weiliang Xiao; xwltc123@163.com

Received 5 May 2014; Accepted 29 June 2014; Published 22 July 2014

Academic Editor: Robert A. Van Gorder

Copyright (C) 2014 Weiliang Xiao et al. This is an open access article distributed under the Creative Commons Attribution License, which permits unrestricted use, distribution, and reproduction in any medium, provided the original work is properly cited.

We study the Cauchy problem of the fractional Navier-Stokes equations in critical Fourier-Besov spaces $F \dot{B}_{p, q}^{1-2 \beta+3 / p^{\prime}}$. Some properties of Fourier-Besov spaces have been discussed, and we prove a general global well-posedness result which covers some recent works in classical Navier-Stokes equations. Particularly, our result is suitable for the critical case $\beta=1 / 2$. Moreover, we prove the long time decay of the global solutions in Fourier-Besov spaces.

\section{Introduction}

We study the mild solutions to the fractional Navier-Stokes equations in $R^{+} \times R^{3}$ as follows:

$$
\begin{gathered}
u_{t}+\mu(-\Delta)^{\beta} u+(u \cdot \nabla) u+\nabla \pi=0, \quad(t, x) \in R^{+} \times R^{3} \\
\nabla \cdot u=0, \quad(t, x) \in R^{+} \times R^{3} \\
u(0, x)=u_{0}(x), \quad x \in R^{3} .
\end{gathered}
$$

Here $u(t, x)=\left(u_{1}(t, x), u_{2}(t, x), u_{3}(t, x)\right)$ denotes the velocity vector, $\mu>0$ is the viscosity coefficient, and the scalar function $\pi$ denotes the pressure. The initial data $u_{0}$ is a divergence free vector field and the operator $(-\Delta)^{\beta}$ is the Fourier multiplier with symbol $|\xi|^{2 \beta}$.

The fractional Navier-Stokes equations, which are also called generalized Navier-Stokes equations, enjoy an invariance under the scaling

$$
\begin{gathered}
u_{\lambda}(t, x)=\lambda^{2 \beta-1} u\left(\lambda^{2 \beta} t, \lambda x\right), \\
p_{\lambda}(t, x)=\lambda^{4 \beta-2} p\left(\lambda^{2 \beta} t, \lambda x\right), \\
u_{0, \lambda}=\lambda^{2 \beta-1} u_{0}(\lambda x) .
\end{gathered}
$$

We say that a function space is $\beta$-critical for (1) if its norm is invariant under the scaling $u_{0}(x) \rightarrow \lambda^{2 \beta-1} u_{0}(\lambda x)$. There are many examples of critical spaces, for instance, $B M O^{-(2 \beta-1)}$, $\dot{B}^{-(2 \beta-1)}$, and the spaces we will discuss in this paper.

The classical incompressible Navier-Stokes equations (i.e., $\beta=1$ ) have been intensively studied. Leray first [1] introduced the concept of weak solutions and obtained the global existence of weak solutions. Fujita and Kato [2] gave a different approach to study the equations in their equivalent form of integral equations and proved the well-posedness in the space frame $\dot{H}^{1 / 2}$. A series study of mild solutions in different function spaces then arose, for instance, Kato [3] in Lebesgue space $L^{3}\left(R^{3}\right)$, Cannone [4] in Besov space $\dot{B}_{p, \infty}^{-1+3 / p}$, and the important well-posedness in $B M O^{-1}$ by Koch and Tataru [5]. These works naturally lead one to study the wellposedness in the largest critical space $\dot{B}_{\infty, \infty}^{-1}$. In fact, all the above spaces are critical spaces and satisfy the following continuous embeddings in the 3 dimensions:

$$
\dot{H}^{1 / 2} \hookrightarrow L^{3} \hookrightarrow \dot{B}_{p, \infty(p<\infty)}^{-1+3 / p} \hookrightarrow B M O^{-1} \hookrightarrow \dot{B}_{\infty, \infty}^{-1}
$$

However, in the space $\dot{B}_{\infty, \infty}^{-1}$, the Navier-Stokes equations are ill-posedness (see Bourgain and Pavlović [6] and Cheskidov and Shvydkoy [7]). 
As for the generalized case (1), Lions [8] proved the global existence of classical solutions in 3 dimensions when $\beta \geq 5 / 4$ (see also $\mathrm{Wu}$ [9] in $n$ dimensions). For the important case $\beta<5 / 4$, Wu $[10,11]$ studied the well-posedness in $\dot{B}_{p, q}^{1-2 \beta+3 / p}$. Inspired by Xiao [12] in the classical case $(\beta=1), \mathrm{Li}$ and Zhai $[13,14]$ studied (1) in some critical $Q$-type spaces for $\beta \in(1 / 2,1)$, and Zhai [15] showed the well-posedness in $B M O^{-(2 \beta-1)}$ when $\beta \in(1 / 2,1)$. For the biggest critical space $\dot{B}_{\infty, \infty}^{-(2 \beta-1)}$, Yu and Zhai [16] proved the well-posedness when $\beta \in(1 / 2,1)$, Cheskidov and Shvydkoy [17] showed the illposedness when $\beta \in[1,5 / 4)$. Very recently, Deng and Yao [18] studied (1) in Triebel-Lizorkin spaces $\dot{F}_{\alpha, r}^{-\beta}$ and obtained the well-posedness in $\dot{F}_{3 /(\beta-1), 2}^{-\beta}$ and ill-posedness in $\dot{F}_{3 /(\beta-1), r}^{-\beta}$ $(r>2)$ in the case $\beta \in(1,5 / 4)$.

In this paper, we will study (1) in the Fourier-Besov spaces $F \dot{B}_{p, q}^{s}$. We observe that although the Fourier-Besov spaces $F \dot{B}_{p, q}^{s}$ appear in the literature very recently, they have received a lot of attentions in studying Navier-Stokes equations, although sometime people gave these spaces several different names. An early paper by Cannone and Karch [19] worked in the space $\mathscr{P} \mathscr{M}^{a}$, which is in fact the space $F \dot{B}_{\infty, \infty}^{a}$ (see Section 2 for details). Biswas and Swanson [20] studied the Gevrey regularity of Navier-Stokes equations in $F \dot{B}_{p, p}^{2-3 / p}$. Konieczny and Yoneda [21] used $F \dot{B}_{p, q}^{s}$ to study the NavierStokes equations with Coriolis (see also Fang et al. [22]). Lei and Lin [23] proved global existence of mild solutions in $\mathscr{X}^{-1}$, which is in fact equal to the space $F \dot{B}_{1,1}^{-1}$. Cannone and $\mathrm{Wu}$ [24] extended the result in [23] to the Fourier-Herz spaces $\dot{\mathscr{B}}_{q}^{s}$. We may notice that $\dot{\mathscr{B}}_{q}^{s}=F \dot{B}_{1, q}^{-1}$. Also, some properties of solutions in the space $\mathscr{X}^{-1}$ have been studied recently; see Zhang and Yin [25] for the blow-up criterion and Benameur [26] for the long time decay. All the above-mentioned works are involved in the classical Navier-Stokes equations. Those indicate that the Fourier-Besov spaces $F \dot{B}_{p, q}^{s}$ might be good work frames in the study of Navier-Stokes equations. Inspired by these observations, in this paper, we will study generalized Navier-Stokes equations in $F \dot{B}_{p, q}^{s}$. We obtain a global wellposedness result which is more general than those in $[23,24]$. Particularly, our well-posedness is also valid in the critical case $\beta=1 / 2$. Moreover, the long time decay of the solutions in Fourier-Besov spaces is also proved, which fully extends the result of [26].

Throughout this paper, the notation $A \sim B$ means that there exist positive constants $C_{1} \leq C_{2}$ such that $C_{1} A \leq$ $B \leq C_{2} A$. We use $\dot{B}_{p, q}^{s}$ to denote the classical homogenous Besov spaces and $\dot{H}^{s}$ the homogenous Sobolev spaces. Also, $C$ denotes a positive constant which may differ in lines if not being specified; $p^{\prime}$ is the number satisfying $1 / p+1 / p^{\prime}=1$ for $1 \leq p \leq \infty$. The inverse Fourier transform is denoted by $\mathscr{F}^{-1}$.

We organize the paper as follows. In Section 2 we give the definition of Fourier-Besov spaces and discuss some basic properties of these spaces. Our main results are also stated in this section. In Section 3 we prove the global well-posedness and in Section 4 we prove the long time decay property.

\section{Preliminaries and Main Results}

We first introduce the definition of Fourier-Besov spaces in $n$ dimensions. Let $\varphi \in C_{c}^{\infty}\left(R^{n}\right)$ be a radial real-valued smooth function such that $0 \leq \varphi(\xi) \leq 1$ and

$$
\begin{gathered}
\operatorname{supp} \varphi \subset\left\{\xi \in R^{n}: \frac{3}{4} \leq|\xi| \leq \frac{8}{3}\right\}, \\
\sum_{j \in Z} \varphi\left(2^{-j} \xi\right)=1,
\end{gathered}
$$$$
\text { for any } \xi \neq 0 \text {. }
$$

We denote $\varphi_{j}(\xi)=\varphi\left(2^{-j} \xi\right)$ and $\mathbb{P}$ the set of all polynomials. The space of tempered distributions is denoted by $S^{\prime}$.

Definition 1. For $s \in R, 1 \leq p, q \leq \infty$, set

$$
\|f\|_{F \dot{B}_{p, q}^{s}}= \begin{cases}\left(\sum_{j \in Z} 2^{j s q}\left\|\varphi_{j} \widehat{f}\right\|_{L^{p}}^{q}\right)^{1 / q}, & q<\infty ; \\ \sup _{j \in Z} 2^{j s}\left\|\varphi_{j} \hat{f}\right\|_{L^{p}}, & q=\infty .\end{cases}
$$

One defines the homogeneous Fourier-Besov space $F \dot{B}_{p, q}^{s}$ as

$$
F \dot{B}_{p, q}^{s}=\left\{f \in \frac{S^{\prime}}{\mathbb{P}}:\|f\|_{F \dot{B}_{p, q}^{s}}<\infty\right\} .
$$

We see that the Fourier-Besov spaces are defined in a similar way with the classical homogeneous Besov spaces, but there are lack of the inverse Fourier transform. This allows us to derive estimates by Hölder's inequality directly, instead of using Bernstein's inequality. Now we explain that FourierBesov spaces contain some known spaces applied in studying Navier-Stokes equations.

Cannone and Karch [19] introduced the spaces $\mathscr{P} \mathscr{M}^{a}$ as follows:

$$
\begin{aligned}
\mathscr{P} \mathscr{M}^{a}= & \left\{v \in S^{\prime}: \widehat{v} \in L_{\text {loc }}^{1},\right. \\
& \left.\|v\|_{\mathscr{P} \mathscr{M}^{a}}=\operatorname{esssup}_{\xi \in R^{n}}|\xi|^{a}|\widehat{v}(\xi)|<\infty\right\} .
\end{aligned}
$$

We easily see that $\mathscr{P} \mathscr{M}^{a}=F \dot{B}_{\infty, \infty}^{a}$.

The norm of Fourier-Herz spaces $\dot{\mathscr{B}}_{q}^{s}$ in [24] is defined as

$$
\|f\|_{\dot{\mathscr{B}}_{q}^{s}}= \begin{cases}\left(\sum_{j \in Z} 2^{j s q}\left\|\varphi_{j} \hat{f}\right\|_{L^{1}}^{q}\right)^{1 / q}, & q<\infty ; \\ \sup _{j \in Z} 2^{j s}\left\|\varphi_{j} \hat{f}\right\|_{L^{1}}, & q=\infty .\end{cases}
$$

Obviously, we have $\dot{\mathscr{B}}_{q}^{s}=F \dot{B}_{1, q}^{s}$.

The space $\mathscr{X}^{-1}$ introduced by Lei and Lin [23] is

$$
\mathscr{X}^{-1}=\left\{f \in S^{\prime}\left(R^{n}\right): \int_{R^{n}}|\xi|^{-1}|\widehat{f}| d \xi<\infty\right\} .
$$


We claim that $\mathscr{X}^{-1}=F \dot{B}_{1,1}^{-1}$. This fact can be seen by the following proposition (proof in [21]).

Proposition 2. Define the spaces $\mathscr{X}^{s}$ as

$$
\mathscr{X}^{s}=\left\{f \in \frac{S^{\prime}}{\mathbb{P}}:\left(\int_{R^{n}}|\xi|^{s p}|\widehat{f}|^{p} d \xi\right)^{1 / p}<\infty\right\} .
$$

Then one has $\mathscr{X}^{s}=F \dot{B}_{p, p}^{s}$ and the norms are equivalent:

$$
\|f\|_{F \dot{B}_{p, p}^{s}} \sim\left(\int_{R^{n}}|\xi|^{s p}|\hat{f}|^{p} d \xi\right)^{1 / p}
$$

We discuss some inclusion relationships in $F \dot{B}_{p, q}^{s}$.

Proposition 3. Let $s \in R, 1 \leq p, q \leq \infty$. One has the follwoing.

(1) If $p=2$, then $F \dot{B}_{2, q}^{s}=\dot{B}_{2, q}^{s}$.

(2) If $p<2$, then $F \dot{B}_{p, q}^{s} \subset \dot{B}_{p^{\prime}, q}^{s}$.

(3) If $q_{1} \leq q_{2}$, then $F \dot{B}_{p, q_{1}}^{s} \subset F \dot{B}_{p, q_{2}}^{s}$.

(4) If $1 \leq q \leq \infty, 1 \leq p_{1} \leq p_{2} \leq \infty$, and $s_{1}, s_{2} \in R$ satisfy $s_{1}+n / p_{1}=s_{2}+n / p_{2}$, then

$$
F \dot{B}_{p_{2}, q}^{s_{2}} \subset F \dot{B}_{p_{1}, q}^{s_{1}}
$$

(5) If $s=(1-\theta) s_{1}+\theta s_{2}, 1 / p=(1-\theta) / p_{1}+(1-\theta) / p_{2}$, and $1 / q=(1-\theta) / q_{1}+(1-\theta) / q_{2}$ for $0 \leq \theta \leq 1$, then

$$
\|f\|_{F \dot{B}_{p, q}^{s}} \leq\|f\|_{F \dot{B}_{p_{1}, q_{1}}^{s_{1}}}^{1-\theta}\|f\|_{F \dot{B}_{p_{2}, q_{2}}^{s_{2}}}^{\theta} .
$$

Proof. (1) is a consequence of Plancherel's identity, and Hausdorff-Young's inequality gives (2). Equation (3) is just the inclusion $l^{q_{1}} \subset l^{q_{2}}$ for $1 \leq q_{1} \leq q_{2} \leq \infty$. To conclude (4), we use Hölder's inequality to get

$$
\left\|\varphi_{j} \widehat{f}\right\|_{L^{p_{1}}} \leq C 2^{j n\left(1 / p_{1}-1 / p_{2}\right)}\left\|\varphi_{j} \widehat{f}\right\|_{L^{p_{2}}}
$$

Since $s_{1}$ and $s_{2}$ satisfy $s_{1}+n / p_{1}=s_{2}+n / p_{2}$, we immediately get

$$
2^{j s_{1}}\left\|\varphi_{j} \widehat{f}\right\|_{L^{p_{1}}} \leq C 2^{j s_{2}}\left\|\varphi_{j} \widehat{f}\right\|_{L^{p_{2}}} .
$$

Taking the $l^{q}$-norm on the above inequality we have

$$
\|f\|_{F \dot{B}_{p_{1}, q}^{s_{1}}} \leq C\|f\|_{F \dot{B}_{p_{2}, q}^{s_{2}}} .
$$

To prove (5), we have

$$
\begin{aligned}
\|f\|_{F \dot{B}_{p, q}^{s}} & =\left(\sum_{j} 2^{j s q}\left\|\left|\varphi_{j} \widehat{f}\right|^{1-\theta}\left|\varphi_{j} \widehat{f}\right|^{\theta}\right\|_{L^{p}}^{q}\right)^{1 / q} \\
& \leq\left(\sum_{j} 2^{j(1-\theta) s_{1} q}\left\|\varphi_{j} \hat{f}\right\|_{L^{p_{1}}}^{(1-\theta) q} 2^{j \theta s_{2} q}\left\|\varphi_{j} \widehat{f}\right\|_{L^{p_{2}}}^{\theta q}\right)^{1 / q} \\
& \leq\|f\|_{F \dot{B}_{p_{1}, q_{1}}^{s_{1}}}^{1-\theta}\|f\|_{F \dot{B}_{p_{2}, q_{1}}^{s_{2}}}^{\theta} .
\end{aligned}
$$

Now we are ready to state our main results. From now on in this paper we take the dimension $n=3$.

Definition 4. Let $s \in R, 1 \leq p, q \leq \infty$, and $I=[0, T), T \in$ $(0, \infty]$. The space-time norm is defined on $f(t, x)$ by

$$
\|f(t, x)\|_{\mathscr{L}^{r}\left(I ; F \dot{B}_{p, q}^{s}\right)}:=\left(\sum_{j \in Z} 2^{j s q}\left\|\varphi_{j} \widehat{f}\right\|_{L^{r}\left(I ; L^{p}\right)}^{q}\right)^{1 / q} .
$$

Our first result is on the well-posedness of (1).

Theorem 5. Let $1 \leq p, q \leq \infty$, and $1 / 2<\beta<\min \{1+$ $\left.3 / p^{\prime}, 5 / 2\right\}$. Then there exists a constant $C_{0}=C_{0}(\beta, p, q)$ such that, for any $u_{0} \in F \dot{B}_{p, q}^{1-2 \beta+3 / p^{\prime}}$ with $\nabla \cdot u_{0}=0$ satisfying

$$
\left\|u_{0}\right\|_{F \dot{B}_{p, q}^{1-2 \beta+3 / p^{\prime}}}<C_{0} \mu,
$$

the Cauchy problem (1) admits a unique global mild solution $u$ and

$$
u \in \mathscr{C}\left([0, \infty) ; F \dot{B}_{p, q}^{1-2 \beta+3 / p^{\prime}}\right) \cap \mathscr{L}^{1}\left([0, \infty) ; F \dot{B}_{p, q}^{1+3 / p^{\prime}}\right),
$$

and it satisfies

$$
\begin{aligned}
& \|u\|_{\mathscr{L}^{\infty}\left([0, \infty) ; \dot{B}_{p, q}^{1-2 \beta+3 / p^{\prime}}\right)}+\mu\|u\|_{\mathscr{L}^{1}\left([0, \infty) ; F \dot{B}_{p, q}^{1+3 / p^{\prime}}\right)} \\
& \quad \leq 2\left(1+\left(\frac{16}{9}\right)^{\beta}\right)\left\|u_{0}\right\|_{F \dot{B}_{p, q}^{1-2 \beta+3 / p^{\prime}}} .
\end{aligned}
$$

Particularly, our result also holds in the critical case $q=1$ and $\beta=1 / 2$.

Remark 6. We emphasize that the case $\beta=1 / 2$ is important, since it is also the critical case for the fractional Navier-Stokes equations. Note that when $\beta=1 / 2$, the function spaces we work on are $F \dot{B}_{p, 1}^{3 / p^{\prime}}$. All these spaces are embedded into $F \dot{B}_{1,1}^{0}$, which is the space that consists of all functions whose Fourier transforms are in $L^{1}$ (see Proposition 2).

Remark 7. Note that $F \dot{B}_{p, q}^{1-2 \beta+3 / p^{\prime}} \subset \dot{B}_{\infty, \infty}^{-2 \beta-1}$ by Proposition 3 and the space $F \dot{B}_{p, q}^{1-2 \beta+3 / p^{\prime}}$ are also critical spaces. In fact, for $u_{0, \lambda}=\lambda^{2 \beta-1} u_{0}(\lambda x)$, we have $\widehat{u_{0, \lambda}}=\lambda^{2 \beta-4} \widehat{u_{0}}\left(\lambda^{-1} \xi\right)$. Set

$$
g_{j}(\xi):=\varphi\left(2^{-j+\left[\log _{2} \lambda\right]-\log _{2} \lambda} \xi\right) \widehat{u_{0, \lambda}}(\xi) \text {. }
$$

Then we have

$$
\begin{aligned}
2^{j\left(1-2 \beta+3 / p^{\prime}\right)}\left\|g_{j}\right\|_{L^{p}} & 2^{j\left(1-2 \beta+3 / p^{\prime}\right)}\left\|\varphi\left(2^{-j+\left[\log _{2} \lambda\right]-\log _{2} \lambda} \xi\right) \lambda^{2 \beta-4} \widehat{u_{0}}\left(\lambda^{-1} \xi\right)\right\|_{L^{p}} \\
= & 2^{\left(\left[\log _{2} \lambda\right]-\log _{2} \lambda\right)\left(1-2 \beta+3 / p^{\prime}\right)} \\
& \times 2^{\left(j-\left[\log _{2} \lambda\right]\right)\left(1-2 \beta+3 / p^{\prime}\right)}\left\|\varphi\left(2^{-j+\left[\log _{2} \lambda\right]} \eta\right) \widehat{u_{0}}(\eta)\right\|_{L^{p}} \\
\sim & 2^{\left(j-\left[\log _{2} \lambda\right]\right)\left(1-2 \beta+3 / p^{\prime}\right)}\left\|\varphi\left(2^{-j+\left[\log _{2} \lambda\right]} \eta\right) \widehat{u_{0}}(\eta)\right\|_{L^{p}}
\end{aligned}
$$


This implies that

$$
\left(\sum_{j \in Z} 2^{j q\left(1-2 \beta+3 / p^{\prime}\right)}\left\|g_{j}\right\|_{L^{p}}^{q}\right)^{1 / q} \sim\left\|u_{0}\right\|_{F \dot{B}_{p, q}^{1-2 \beta+3 / p^{\prime}}} .
$$

On the other hand, by

$$
\varphi_{j}(\xi) \widehat{u_{0, \lambda}}(\xi)=\sum_{|k-j| \leq 2} \varphi_{j}(\xi) g_{k}(\xi)
$$

we can easily deduce that

$$
\left\|u_{0, \lambda}\right\|_{F \dot{B}_{p, q}^{1-2 \beta+3 / p^{\prime}}} \sim\left(\sum_{j \in Z} 2^{j q\left(1-2 \beta+3 / p^{\prime}\right)}\left\|g_{j}\right\|_{L^{p}}^{q}\right)^{1 / q} .
$$

Unfortunately, Theorem 5 is not suitable for the case $\beta=$ $1, p=1$, in which similar existence has been proved by Cannone and $\mathrm{Wu}$ [24]. To address this case, we also get the following theorem.

Theorem 8. Let $1 \leq p \leq q \leq 2$ and $\beta \in\left(1 / 2,1+3 / 2 p^{\prime}\right]$. Then there exists a constant $C_{0}=C_{0}(\beta, p, q)$ such that, for any $u_{0} \in F \dot{B}_{p, q}^{1-2 \beta+3 / p^{\prime}}$ with $\nabla \cdot u_{0}=0$ satisfying

$$
\left\|u_{0}\right\|_{F \dot{B}_{p, q}^{1-2 \beta+3 / p^{\prime}}}<C_{0} \mu
$$

the Cauchy problem (1) admits a unique global mild solution $u$ and

$$
u \in \mathscr{C}\left([0, \infty) ; F \dot{B}_{p, q}^{1-2 \beta+3 / p^{\prime}}\right) \cap \mathscr{L}^{1}\left([0, \infty) ; F \dot{B}_{p, q}^{1+3 / p^{\prime}}\right)
$$

and it satisfies

$$
\begin{aligned}
& \|u\|_{\mathscr{L}^{\infty}\left([0, \infty) ; F \dot{B}_{p, q}^{1-2 \beta+3 / p^{\prime}}\right)}+\mu\|u\|_{\mathscr{L}^{1}\left([0, \infty) ; F \dot{B}_{p, q}^{1+3 / p^{\prime}}\right)} \\
& \quad \leq 2\left(1+\left(\frac{16}{9}\right)^{\beta}\right)\left\|u_{0}\right\|_{F \dot{B}_{p, q}^{1-2 \beta+3 / p^{\prime}}} \cdot
\end{aligned}
$$

Particularly, our result also holds in the critical case $p=q=1$ and $\beta=1 / 2$.

Remark 9. In comparison with Theorem 5, although we have a limitation $1 \leq p \leq q \leq 2$, the regularity index $\beta$ in Theorem 10 lies in a larger interval when $p=1$.

Our third result is on the decay property of the global solutions

Theorem 10. Let $1 \leq p \leq q \leq 2$ and $\beta \in(5 / 6,1]$. Assume that $u \in C\left([0, \infty) ; F \dot{B}_{p, q}^{1-2 \beta+3 / p^{\prime}}\right)$ is a global solution of (1). One has

$$
\limsup _{t \rightarrow \infty}\|u(t)\|_{F \dot{B}_{p, q}^{1-2 \beta+3 / p^{\prime}}}=0 .
$$

Remark 11. Recently, Benameur [26] obtained the same property in the space $\mathscr{X}^{-1}=F \dot{B}_{1,1}^{-1}$ for the classical NavierStokes equations $(\beta=1)$. Our result improves and extends his result.

\section{The Well-Posedness}

First, we study the linear estimates of (1). For this purpose we consider the dissipative equation:

$$
\begin{gathered}
u_{t}+\mu(-\Delta)^{\beta} u=F(t, x), \quad(t, x) \in R^{+} \times R^{3} ; \\
u(x, 0)=u_{0}(x), \quad x \in R^{3} .
\end{gathered}
$$

It is easy to see that the equivalent integral equation of (31) is

$$
u(t, x)=e^{-\mu t(-\Delta)^{\beta}} u_{0}-\int_{0}^{t} e^{-\mu(t-\tau)(-\Delta)^{\beta}} F(\tau, x) d \tau .
$$

By taking $F(t, x)=0$ or $u_{0}(x)=0$, we obtain the linear term or the nonlinear term of the equation, respectively. This indicates that the following lemma is very useful in our later proof.

Lemma 12 (linear estimate). Let $I=[0, T), 0<T \leq \infty$, $s \in R, 1 \leq p$, and $q \leq \infty$. Assume that $u_{0} \in F \dot{B}_{p, q}^{s}$ and $F \in$ $\mathscr{L}^{1}\left(I ; F \dot{B}_{p, q}^{s}\right)$. Then the solution $u(t, x)$ to the Cauchy problem (31) satisfies

$$
\begin{aligned}
& \|u\|_{\mathscr{L}^{\infty}\left(I ; F \dot{B}_{p, q}^{s}\right)}+\mu\|u\|_{\mathscr{L}^{1}\left(I ; F \dot{B}_{p, q}^{s+2 \beta}\right)} \\
& \quad \leq\left(1+\left(\frac{16}{9}\right)^{\beta}\right)\left(\left\|u_{0}\right\|_{F \dot{B}_{p, q}^{s}}+\|F\|_{\mathscr{L}^{1}\left(I ; \dot{B}_{p, q}^{s}\right)}\right) .
\end{aligned}
$$

Proof. By taking the Fourier transform we have

$$
\partial_{t} \widehat{u}+\mu|\xi|^{2 \beta} \widehat{u}=\widehat{F}
$$

Multiplying $\varphi_{j}$ and taking the $L^{p}$-norm on both sides,

$$
\frac{d}{d t}\left\|\widehat{u}_{j}\right\|_{L^{p}}+\left(\frac{9}{16}\right)^{\beta} \mu 2^{2 \beta j}\left\|\widehat{u}_{j}\right\|_{L^{p}} \leq\left\|\widehat{F}_{j}\right\|_{L^{p}}
$$

where we denote $\widehat{u}_{j}=\varphi_{j} \widehat{u}$. Integrating with respect to time on $[0, t)$, we get

$$
\left\|\widehat{u}_{j}\right\|_{L^{p}}+\left(\frac{9}{16}\right)^{\beta} \mu 2^{2 \beta j}\left\|\widehat{u}_{j}\right\|_{L^{1}\left(0, t ; L^{p}\right)} \leq\left\|\widehat{u_{0} j}\right\|_{L^{p}}+\left\|\widehat{F}_{j}\right\|_{L^{1}\left(I ; L^{p}\right)} .
$$

By the definition of $F \dot{B}_{p, q}^{s}$ and the triangle inequality for $l^{q}$, it is easy to obtain our desired inequality.

Next we consider the bilinear estimate, which is the key estimate in solving the Navier-Stokes equations.

Lemma 13 (bilinear estimate). Let $1 \leq p, q \leq \infty$, and $1 / 2<$ $\beta<\min \left\{1+3 / p^{\prime}, 5 / 2\right\}$ and set

$$
X=\mathscr{L}^{\infty}\left(I ; F \dot{B}_{p, q}^{1-2 \beta+3 / p^{\prime}}\right) \cap \mathscr{L}^{1}\left(I ; F \dot{B}_{p, q}^{1+3 / p^{\prime}}\right)
$$

with the norm

$$
\|u\|_{X}=\|u\|_{\mathscr{L}^{\infty}\left(I ; F \dot{B}_{p, q}^{1-2 \beta+3 / p^{\prime}}\right)}+\mu\|u\|_{\mathscr{L}^{1}\left(I ; F \dot{B}_{p, q}^{1+3 / p^{\prime}}\right)} .
$$


Then there exists some constant $C=C(\beta, p, q)>0$ depending on $\beta, p$, and $q$ such that

$$
\|\nabla \cdot(u \otimes v)\|_{\mathscr{L}^{1}\left(I ; F \dot{B}_{p, q}^{1-2 \beta+3 / p^{\prime}}\right)} \leq C \mu^{-1}\|u\|_{X}\|v\|_{X}
$$

Particularly, it is true for the cases $q=1$ and $\beta=1 / 2$.

Proof. We will use the technique of the paraproduct. Set

$$
\begin{aligned}
\dot{\Delta}_{j} u & =\left(\mathscr{F}^{-1} \varphi_{j}\right) * u, \\
\dot{S}_{j} & =\sum_{k \leq j-1} \dot{\Delta}_{k} u, \\
\tilde{\dot{\Delta}}_{j} u & =\sum_{|k-j| \leq 1} \dot{\Delta}_{k} u,
\end{aligned}
$$

$$
\text { for } \forall j \in Z \text {. }
$$

By Bony's decomposition, we have for fixed $j$

$$
\begin{aligned}
\dot{\Delta}_{j}(u v)= & \sum_{|k-j| \leq 4} \dot{\Delta}_{j}\left(\dot{S}_{k-1} u \dot{\Delta}_{k} v\right) \\
& +\sum_{|k-j| \leq 4} \dot{\Delta}_{j}\left(\dot{S}_{k-1} v \dot{\Delta}_{k} u\right) \\
& +\sum_{k \geq j-3} \dot{\Delta}_{j}\left(\dot{\Delta}_{k} u \tilde{\dot{\Delta}}_{k} v\right) \\
:= & I_{j}+I I_{j}+I I I_{j} .
\end{aligned}
$$

For simplicity, we can view $\nabla \cdot(u \otimes v)$, as the first derivative of two scale functions $u, v$. Consider

$$
\begin{aligned}
\|\partial(u v)\|_{\mathscr{L}^{1}\left(I: F \dot{B}_{p, q}^{1-2 \beta+3 / p^{\prime}}\right)} & \\
\leq \frac{8}{3} & \left(\sum_{j} 2^{\left(1-2 \beta+3 / p^{\prime}\right) j q} 2^{j q}\left\|\widehat{\dot{\Delta}_{j}(u v)}\right\|_{L^{1}\left(I ; L^{p}\right)}^{q}\right)^{1 / q} \\
\leq \frac{8}{3} & {\left[\left(\sum_{j} 2^{\left(2-2 \beta+3 / p^{\prime}\right) j q}\left\|\widehat{I}_{j}\right\|_{L^{1}\left(I ; L^{p}\right)}^{q}\right)^{1 / q}\right.} \\
+ & \left(\sum_{j} 2^{\left(2-2 \beta+3 / p^{\prime}\right) j q}\left\|\widehat{I I}_{j}\right\|_{L^{1}\left(I ; L^{p}\right)}^{q}\right)^{1 / q} \\
+ & \left.\left(\sum_{j} 2^{\left(2-2 \beta+3 / p^{\prime}\right) j q}\left\|\widehat{I I I}_{j}\right\|_{L^{1}\left(I ; L^{p}\right)}^{q}\right)^{1 / q}\right] .
\end{aligned}
$$

The terms $I_{j}$ and $I I_{j}$ are symmetrical. Using Young's inequality and Hölder's inequality we have

$$
\begin{aligned}
\left\|\widehat{I}_{j}\right\|_{L^{1}\left(I ; L^{p}\right)} \leq & \sum_{|k-j| \leq 4}\left\|\dot{S}_{k-1} \widehat{u \dot{\Delta}_{k}} v\right\|_{L^{1}\left(I ; L^{p}\right)} \\
\leq & \sum_{|k-j| \leq 4}\left\|\widehat{v}_{k}\right\|_{L^{1}\left(I ; L^{p}\right)} \sum_{l \leq k-2}\left\|\widehat{u}_{l}\right\|_{L^{\infty}\left(I ; L^{1}\right)} \\
\leq & \sum_{|k-j| \leq 4}\left\|\widehat{v}_{k}\right\|_{L^{1}\left(I ; L^{p}\right)} \\
& \times\left(\sum_{l \leq k-2} 2^{(1-2 \beta) l q}\left\|\widehat{u}_{l}\right\|_{L^{\infty}\left(I ; L^{1}\right)}^{q}\right)^{1 / q} \\
& \times\left(\sum_{l \leq k-2} 2^{(2 \beta-1) l q^{\prime}}\right)^{1 / q^{\prime}} \\
\leq & C \sum_{|k-j| \leq 4} 2^{(2 \beta-1) k}\left\|\widehat{v}_{k}\right\|_{L^{1}\left(I ; L^{p}\right)}\|u\|_{\mathscr{L}^{\infty}\left(I ; F \dot{B}_{1, q}^{1-2 \beta}\right)} .
\end{aligned}
$$

Using the conclusion $F \dot{B}_{p, q}^{1-2 \beta+3 / p^{\prime}} \subset F \dot{B}_{1, q}^{1-2 \beta}$, we have

$$
\begin{aligned}
& \left(\sum_{j} 2^{\left(2-2 \beta+3 / p^{\prime}\right) j q}\left\|\widehat{I}_{j}\right\|_{L^{1}\left(I ; L^{p}\right)}^{q}\right)^{1 / q} \\
& \quad \leq C\|u\|_{\mathscr{L}^{\infty}\left(I ; F \dot{B}_{p, q}^{1-2 \beta+3 / p^{\prime}}\right)}\|v\|_{\mathscr{L}^{1}\left(I ; F \dot{B}_{p, q}^{1+3 / p^{\prime}}\right)}
\end{aligned}
$$

In a similar way we can prove that

$$
\begin{aligned}
& \left(\sum_{j} 2^{\left(2-2 \beta+3 / p^{\prime}\right) j q}\left\|\widehat{I I}_{j}\right\|_{L^{1}\left(I ; L^{p}\right)}^{q}\right)^{1 / q} \\
& \leq C\|v\|_{\mathscr{L}^{\infty}\left(I ; F \dot{B}_{p, q}^{1-2 \beta+3 / p^{\prime}}\right)}\|u\|_{\mathscr{L}^{1}\left(I ; F \dot{B}_{p, q}^{1+3 / p^{\prime}}\right)}
\end{aligned}
$$

For the remaining term, we first consider the case $p \leq 2$ in which $\beta<1+3 / p^{\prime}$. By Hölder's inequality with $1 / p=1 / p^{\prime}+$ $1 / p-1 / p^{\prime}$ and by Young's inequality with $1+1 / p-1 / p^{\prime}=$ $1 / p+1 / p$, we have

$$
\begin{aligned}
& 2^{\left(2-2 \beta+3 / p^{\prime}\right) j}\|\widehat{I I I} j\|_{L^{1}\left(I ; L^{p}\right)} \\
& \leq C \sum_{k \geq j-3} 2^{\left(2-2 \beta+3 / p^{\prime}\right) j} 2^{\left(3 / p^{\prime}\right) j}\left\|\widehat{u_{k}} * \sum_{|l-k| \leq 1} \widehat{v_{l}}\right\|_{L^{1}\left(I ; L^{p p^{\prime} /\left(p^{\prime}-p\right)}\right)} \\
& \leq C \sum_{k \geq j-3} 2^{\left(2-2 \beta+6 / p^{\prime}\right) j}\left\|\widehat{u_{k}}\right\|_{L^{1}\left(I ; L^{p}\right)} \sum_{|l-k| \leq 1}\left\|\widehat{v_{l}}\right\|_{L^{\infty}\left(I ; L^{p}\right)} \\
& \leq C \sum_{k \geq j-3} 2^{\left(2-2 \beta+6 / p^{\prime}\right)(j-k)} 2^{\left(1-2 \beta+3 / p^{\prime}\right) k}\left\|\widehat{u_{k}}\right\|_{L^{\infty}\left(I ; L^{p}\right)} \\
& \quad \times \sum_{|l-k| \leq 1} 2^{\left(1+3 / p^{\prime}\right) l}\left\|\widehat{v_{l}}\right\|_{L^{1}\left(I ; L^{p}\right)} .
\end{aligned}
$$


When $q>2$, we take $l^{q}$-norm of both sides of (46) and use Young's inequality with $1+1 / q=1 / q^{\prime}+2 / q$ to get

$$
\begin{gathered}
\left(\sum_{j} 2^{\left(2-2 \beta+3 / p^{\prime}\right) j q}\|\widehat{I I I} j\|_{L^{1}\left(I ; L^{p}\right)}^{q}\right)^{1 / q} \\
\leq C\left\|2^{\left(1-2 \beta+3 / p^{\prime}\right) k}\right\| \widehat{u_{k}} \|_{L^{\infty}\left(I ; L^{p}\right)} \\
\quad \times \sum_{|l-k| \leq 1} 2^{\left(1+3 / p^{\prime}\right) l}\left\|\widehat{v}_{l}\right\|_{L^{1}\left(I ; L^{p}\right)} \|_{q^{q / 2}(k)} \\
\leq C\|u\|_{\mathscr{L}^{\infty}\left(I ; F \dot{B}_{p, q}^{1-2 \beta+3 / p^{\prime}}\right)}\|v\|_{\mathscr{L}^{1}\left(I ; F \dot{B}_{p, q}^{1+3 / p^{\prime}}\right)} .
\end{gathered}
$$

When $q \leq 2$, since $F \dot{B}_{p, q}^{1+3 / p^{\prime}} \subset F \dot{B}_{p, q^{\prime}}^{1+3 / p^{\prime}}$, we take $l^{q}$-norm of both sides of (46) and use Young's inequality with $1+1 / q=$ $1+1 / q$ to get

$$
\begin{gathered}
\left(\sum_{j} 2^{\left(2-2 \beta+3 / p^{\prime}\right) j q}\|\widehat{I I I} j\|_{L^{1}\left(I: L^{p}\right)}^{q}\right)^{1 / q} \\
\leq C\left\|2^{\left(1-2 \beta+3 / p^{\prime}\right) k}\right\| \widehat{u}_{k} \|_{L^{\infty}\left(I ; L^{p}\right)} \\
\quad \times \sum_{|l-k| \leq 1} 2^{\left(1+3 / p^{\prime}\right) l}\left\|\widehat{v_{l}}\right\|_{L^{1}\left(I ; L^{p}\right)} \|_{l^{1}(k)} \\
\leq C\|u\|_{\mathscr{L}^{\infty}\left(I ; F \dot{B}_{p, q}^{1-2 \beta+3 / p^{\prime}}\right)}\|v\|_{\mathscr{L}^{1}\left(I ; F \dot{B}_{p, q^{\prime}}^{1+3 / p^{\prime}}\right)} \\
\leq C\|u\|_{\mathscr{L}^{\infty}\left(I ; F \dot{B}_{p, q}^{1-2 \beta+3 / p^{\prime}}\right)}\|v\|_{\mathscr{L}^{1}\left(I ; F \dot{B}_{p, q}^{1+3 / p^{\prime}}\right)}
\end{gathered}
$$

Next we consider the case $p>2$ and hence $\beta \leq 5 / 2$. By Hölder's inequality we have

$$
\begin{aligned}
& 2^{\left(2-2 \beta+3 / p^{\prime}\right) j}\|\widehat{I I I}\|_{L^{1}\left(I ; L^{p}\right)} \\
& \leq C \sum_{k \geq j-3} 2^{\left(2-2 \beta+3 / p^{\prime}\right) j} 2^{(3 / p) j}\left\|\widehat{u_{k}} * \sum_{|l-k| \leq 1} \widehat{v_{l}}\right\|_{L^{1}(I} \\
& \leq C \sum_{k \geq j-3} 2^{(5-2 \beta) j}\left\|\widehat{u_{k}}\right\|_{L^{\infty}\left(I ; L^{p^{\prime}}\right)} \sum_{|l-k| \leq 1}\left\|\widehat{v_{l}}\right\|_{L^{1}\left(I ; L^{p}\right)} \\
& \leq C \sum_{k \geq j-3} 2^{(5-2 \beta)(j-k)} 2^{\left(1-2 \beta+3 / p^{\prime}\right) k}\left\|\widehat{u_{k}}\right\|_{L^{\infty}\left(I ; L^{p}\right)} \\
& \quad \times \sum_{|l-k| \leq 1} 2^{\left(1+3 / p^{\prime}\right) l}\left\|\widehat{v_{l}}\right\|_{L^{1}\left(I: L^{p}\right)} .
\end{aligned}
$$

Following the same steps as in the case $p \leq 2$, we obtain the same estimate for $p>2$. Collecting the above estimates we finish our proof.

Next we introduce an abstract lemma on the existence of fixed point solutions $[16,24]$.
Lemma 14. Let $X$ be a Banach space with norm $\|\cdot\|_{X}$ and let $B: X \times X \mapsto X$ be a bounded bilinear operator satisfying

$$
\|B(u, v)\|_{X} \leq \eta\|u\|_{X}\|v\|_{X}
$$

for all $u, v \in X$ and a constant $\eta>0$. Then for any fixed $y \in X$ satisfying $\|y\|_{X}<\epsilon<1 / 4 \eta$, the equation $x:=$ $y+B(x, x)$ has a solution $\bar{x}$ in $X$ such that $\|\bar{x}\|_{X} \leq 2\|y\|_{X}$. Also, the solution is unique in $\bar{B}(0,2 \epsilon)$. Moreover, the solution depends continuously on $y$ in the sense that if $\left\|y^{\prime}\right\|_{X}<\epsilon$, $x^{\prime}=y^{\prime}+B\left(x^{\prime}, x^{\prime}\right)$, and $\left\|x^{\prime}\right\|_{X}<2 \epsilon$, then

$$
\left\|\bar{x}-x^{\prime}\right\|_{X} \leq \frac{1}{1-4 \epsilon \eta}\left\|y-y^{\prime}\right\|_{X}
$$

This lemma allows us to solve the Cauchy problem (1) with bounded bilinear form and small data. The mild solution of (1) is the solution to the equivalent integral form:

$$
\begin{aligned}
u(t, x)= & e^{-\mu t(-\Delta)^{\beta}} u_{0}-\int_{0}^{t} e^{-\mu(t-\tau)(-\Delta)^{\beta}} \mathscr{P} \nabla \\
& \cdot(u \otimes u)(\tau, x) d \tau \\
= & e^{-\mu t(-\Delta)^{\beta}} u_{0}+B(u, u)
\end{aligned}
$$

where $\mathscr{P}=I+\nabla(-\Delta)^{-1}$ div is the Leray-Hopf projector. To make $B(u, v)$ become a bilinear form, we simply take $(1 / 2) u \otimes$ $v+(1 / 2) v \otimes u$ instead of $u \otimes v$ in the integral.

Proof of Theorem 5. We begin with the bilinear operator $B(u, v)$. Observing that $B(u, v)$ can be viewed as the solution to the dissipative equation (31) with $u_{0}=0, F=-\mathscr{P} \nabla \cdot(u \otimes v)$. Thus we can use Lemma 12 with $s=1-2 \beta+3 / p^{\prime}$ and Lemma 13 to obtain

$$
\begin{aligned}
& \|B(u, v)\|_{X} \\
& \quad \leq\left(1+\left(\frac{16}{9}\right)^{\beta}\right)\|-\mathscr{P} \nabla \cdot(u \otimes v)\|_{\mathscr{L}^{1}\left(I ; F \dot{B}_{p, q}^{1-2 \beta+3 / p^{\prime}}\right)} \\
& \quad \leq\left(1+\left(\frac{16}{9}\right)^{\beta}\right) C \mu^{-1}\|u\|_{X}\|v\|_{X} .
\end{aligned}
$$

By Lemma 14 we know that if $\left\|e^{-\mu t(-\Delta)^{\beta}} u_{0}\right\|_{X}<R$ with $R=$ $\mu / 4\left(1+(16 / 9)^{\beta}\right) C$, then $(52)$ has a unique solution in $B(0,2 R)$, where

$$
B(0,2 R):=\left\{x \in X:\|x\|_{X} \leq 2 R\right\} .
$$

Now we need to derive $\left\|e^{-\mu t(-\Delta)^{\beta}} u_{0}\right\|_{X}<R$. Similarly, $e^{-\mu t(-\Delta)^{\beta}} u_{0}$ is the solution to the dissipative equation (31) with $u_{0}=u_{0}$ and $F=0$. By Lemma 12 we obtain

$$
\left\|e^{-\mu t(-\Delta)^{\beta}} u_{0}\right\|_{X} \leq\left(1+\left(\frac{16}{9}\right)^{\beta}\right)\left\|u_{0}\right\|_{F \dot{B}_{p, q}^{1-2 \beta+3 / p^{\prime}}} .
$$


Thus we conclude that if $\left\|u_{0}\right\|_{F B_{p, q}^{1-2 \beta+3 / p^{\prime}}}<C_{0} \mu$ with $C_{0}=$ $\left(4\left(1+(16 / 9)^{\beta}\right)^{2} C\right)^{-1}$, then (52) has a unique global solution $u \in X$ satisfying

$$
\|u\|_{X} \leq 2\left\|e^{-\mu t(-\Delta)^{\beta}} u_{0}\right\|_{X} \leq 2\left(1+\left(\frac{16}{9}\right)^{\beta}\right)\left\|u_{0}\right\|_{F \dot{B}_{p, q}^{1-2 \beta+3 / p^{\prime}}} .
$$

The continuity with respect to time is standard and thus we finish our proof.

Proof of Theorem 8 . The method is the same with the proof of Theorem 5. But we substitute Lemma 13 by the following lemma.

Lemma 15. Let $1 \leq p \leq q \leq 2$ and $\beta \in\left(1 / 2,1+3 / 2 p^{\prime}\right]$ and $X$ is the same as in Lemma 13. Then there exists some constant $C=C(\beta, p, q)>0$ depending on $\beta, p$, and $q$ such that

$$
\|\nabla \cdot(u \otimes v)\|_{\mathscr{L}^{1}\left(I ; F \dot{B}_{p, q}^{1-2 \beta+3 / p^{\prime}}\right)} \leq C \mu^{-1}\|u\|_{X}\|v\|_{X} .
$$

Particularly, it is true for the cases $p=q=1$ and $\beta=1 / 2$.

Proof. The proof is also same with Lemma 13. In fact by Bony's decomposition, we divide $\dot{\Delta}_{j}(u v)$ into three parts $I_{j}, I I_{j}$, and $I I I_{j}$. The parts $I_{j}$ and $I I_{J}$ satisfy the same estimate. Hence it is sufficient to deal with the part III $I_{J}$. In fact when $q \geq p$, we have

$$
\begin{aligned}
& \left(\sum_{j} 2^{\left(2-2 \beta+3 / p^{\prime}\right) j q \| \widehat{I I I}} \|_{L^{1}\left(I L^{p}\right)}^{q}\right)^{1 / q} \\
& \leq\left\|\sum_{k \geq j-3} \int_{I}\right\| 2^{\left(2-2 \beta+3 / p^{\prime}\right) j} \varphi_{j}(\xi) \\
& \quad \times\left[\widehat{u_{k}} * \sum_{|l-k| \leq 1} \widehat{v_{l}}\right]\|\|_{L_{\xi}^{p}} d t \|_{l^{q}(j)} \\
& \leq \sum_{k} \int_{I} \| \int_{R^{3}} 2^{\left(2-2 \beta+3 / p^{\prime}\right) p j} \\
& \quad \times\left|\varphi_{j}(\xi)\right|^{p}\left[\widehat{u_{k}} * \sum_{|l-k| \leq 1} \widehat{v_{l}}\right]^{p} d \xi \|_{l^{q / p}(j \leq k+3)}^{1 / p} d t \\
& \quad \sup _{\xi}\left(\sum_{j} \varphi_{j}(\xi)^{q}\right)^{1 / q} \sum_{k} 2^{\left(2-2 \beta+3 / p^{\prime}\right)(k+3)} \\
& \quad \times \int_{I}\left\|\widehat{u_{k}} * \sum_{|l-k| \leq 1} \widehat{v}_{l}\right\|_{L_{\xi}^{p}} d t
\end{aligned}
$$

$$
\begin{aligned}
\leq & 2^{3\left(2-2 \beta+3 / p^{\prime}\right)} \sum_{k} 2^{(1-2 \beta) k}\left\|\widehat{u_{k}}\right\|_{L^{\infty}\left(I: L^{1}\right)} \\
& \times \sum_{|l-k| \leq 1} 2^{\left(1+3 / p^{\prime}\right)(k-l)} 2^{\left(1+3 / p^{\prime}\right) l}\left\|\widehat{v}_{l}\right\|_{L^{1}\left(I ; L^{p}\right)} \\
\leq & C\|u\|_{\mathscr{L}^{\infty}\left(I ; F \dot{B}_{1, q}^{1-2 \beta}\right)}\|v\|_{\mathscr{L}^{1}\left(I ; F \dot{B}_{p, q}^{1+3 / p^{\prime}}\right)} .
\end{aligned}
$$

In the last inequality we use a similar conclusion with (3) in Proposition 3; that is, $\mathscr{L}^{1}\left(I ; F \dot{B}_{p, q}^{1+3 / p^{\prime}}\right) \subset \mathscr{L}^{1}\left(I ; F \dot{B}_{p, q^{\prime}}^{1+3 / p^{\prime}}\right)$, when $q \in[1,2]$.

\section{The Decay Property}

We introduce some lemmas which have interest in themselves.

Lemma 16. Let $\beta<5 / 4, s>5 / 2-2 \beta$, and $1 \leq p, q \leq 2$. Then we have

$$
\|f\|_{F \dot{B}_{p, q}^{1-2 \beta+3 / p^{\prime}}} \leq C\|f\|_{L^{2}}^{1-(5 / 2-2 \beta) / s}\|f\|_{\dot{H}^{s}}^{(5 / 2-2 \beta) / s} .
$$

Proof. By definition and Hölder's inequality we have

$$
\begin{aligned}
\|f\|_{F \dot{B}_{p, q}^{1-2 \beta+3 / p^{\prime}}} & \left(\sum_{j \in Z} 2^{\left(1-2 \beta+3 / p^{\prime}\right) j q}\left\|\varphi_{j} \hat{f}\right\|_{L^{p}}^{q}\right)^{1 / q} \\
\leq & \left(\sum_{j \in Z} 2^{\left(1-2 \beta+3 / p^{\prime}\right) j q}\left\|\varphi_{j} \widehat{f}\right\|_{L^{2}}^{q} 2^{(3 / p-3 / 2) j q}\right)^{1 / q} \\
\leq & \left(\sum_{j \leq M} 2^{(5 / 2-2 \beta) j q}\left\|\varphi_{j} \widehat{f}\right\|_{L^{2}}^{q}\right)^{1 / q} \\
& +\left(\sum_{j \geq M} 2^{(5 / 2-2 \beta-s) j q} 2^{s j q}\left\|\varphi_{j} \widehat{f}\right\|_{L^{2}}^{q}\right)^{1 / q} \\
\leq & C_{1} 2^{(5 / 2-2 \beta-s) M}\left(\sum_{j \in Z}\left\|\varphi_{j} \widehat{f}\right\|_{L^{2}}^{2}\right)^{1 / 2} \\
& +C_{2} 2^{(5 / 2-2 \beta-s) M}\left(\sum_{j \in Z} 2^{s j q}\left\|\varphi_{j} \hat{f}\right\|_{L^{2}}^{2}\right)^{1 / 2} .
\end{aligned}
$$

Since $\dot{B}_{2,2}^{s}=\dot{H}^{s}$ and $\dot{B}_{2,2}^{0}=L^{2}$ and by Proposition 3, we know that $F \dot{B}_{2,2}^{s}=\dot{B}_{2,2}^{s}$; we finish our proof by taking $M$ such that $2^{M}=\left(\|f\|_{\dot{H}^{s}} /\|f\|_{L^{2}}\right)^{1 / s}$.

Lemma 17. Let $\beta \in(1 / 2,1]$ and $1 \leq p, q \leq 2$. Consider

$$
\|u v\|_{\dot{H}^{1-\beta}} \leq C\|u\|_{L^{2}}\|v\|_{\dot{F}_{\dot{B}_{p, q}}^{1-\beta+3 / p^{\prime}}}+C\|u\|_{\dot{H}^{\beta}}\|v\|_{F \dot{B}_{p, q}^{1-2 \beta+3 / p^{\prime}}} .
$$


Proof. We use the equivalence $\dot{H}^{s}=\dot{B}_{2,2}^{s}=F \dot{B}_{2,2}^{s}$. To conclude the result we only need to show that

$$
\|u v\|_{\dot{H}^{1-\beta}} \leq C\|u\|_{L^{2}}\|v\|_{F \dot{B}_{p, 2}^{1-\beta+3 / p^{\prime}}}+C\|u\|_{\dot{H}^{\beta}}\|v\|_{F \dot{B}_{1,2}^{1-2 \beta}}
$$

since we have the conclusions $F \dot{B}_{p, 2}^{1-2 \beta+3 / p^{\prime}} \subset F \dot{B}_{1,2}^{1-2 \beta}$ and $F \dot{B}_{p, q}^{1-2 \beta+3 / p^{\prime}} \subset F \dot{B}_{p, 2}^{1-2 \beta+3 / p^{\prime}}$ by Proposition 3. The method is similar with the proof of Lemma 13. Consider

$$
\begin{aligned}
\|u v\|_{F \dot{B}_{2,2}^{1-\beta}} \leq & \left(\sum_{j} 2^{(1-\beta) j 2}\left\|\widehat{I}_{j}\right\|_{L^{2}}^{2}\right)^{1 / 2} \\
& +\left(\sum_{j} 2^{(1-\beta) j 2}\left\|\widehat{I I}_{j}\right\|_{L^{2}}^{2}\right)^{1 / 2} \\
& +\left(\sum_{j} 2^{(1-\beta) j 2}\|\widehat{I I I}\|_{L^{2}}^{2}\right)^{1 / 2},
\end{aligned}
$$

where $I_{j}, I I_{j}$, and $I I I_{j}$ are the same with the proof of Lemma 13. Consider

$$
\begin{aligned}
\left\|\widehat{I}_{j}\right\|_{L^{2}} \leq & \sum_{|k-j| \leq 4}\left\|\dot{S}_{k-1} \widehat{u \dot{\Delta}_{k}} v\right\|_{L^{2}} \\
\leq & \sum_{|k-j| \leq 4}\left\|\widehat{v_{k}}\right\|_{L^{2}} \sum_{l \leq k-2}\left\|\widehat{u}_{l}\right\|_{L^{1}} \\
\leq & C \sum_{|k-j| \leq 4}\left\|\widehat{v_{k}}\right\|_{L^{2}} \\
& \times\left(\sum_{l \leq k-2} 2^{(1-2 \beta) l 2}\left\|\widehat{u_{l}}\right\|_{L^{1}}^{2}\right)^{1 / 2} \\
& \times\left(\sum_{l \leq k-2} 2^{(2 \beta-1) l 2}\right)^{1 / 2} \\
\leq & C \sum_{|k-j| \leq 4} 2^{(2 \beta-1) k}\left\|\widehat{v_{k}}\right\|_{L^{2}}\|u\|_{F B_{1,2}^{1-2 \beta}} .
\end{aligned}
$$

Thus we get

$$
\left(\sum_{j} 2^{(1-\beta) j 2}\left\|\widehat{I}_{j}\right\|_{L^{2}}^{2}\right)^{1 / 2} \leq C\|v\|_{\dot{H}^{\beta}}\|u\|_{F \dot{B}_{1,2}^{1-2 \beta}} .
$$

To estimate the term $I I_{j}$, we make a minor modification to get

$$
\begin{aligned}
\|\widehat{I I}\|_{L^{2}} \leq & \sum_{|k-j| \leq 4}\left\|\dot{S}_{k-1} \widehat{v \dot{\Delta}_{k}} u\right\|_{L^{2}} \\
\leq & \sum_{|k-j| \leq 4}\left\|\widehat{u_{k}}\right\|_{L^{p}} \sum_{l \leq k-2}\left\|\widehat{v_{l}}\right\|_{L^{2 p /(3 p-2)}} \\
\leq & C \sum_{|k-j| \leq 4}\left\|\widehat{u_{k}}\right\|_{L^{p}} \\
& \times\left(\sum_{l \leq k-2} 2^{-2 l\left(3 / p^{\prime}\right)}\left\|\widehat{v_{l}}\right\|_{L^{2 p /(3 p-2)}}^{2}\right)^{1 / 2} \\
& \times\left(\sum_{l \leq k-2} 2^{2 l\left(3 / p^{\prime}\right)}\right)^{1 / 2} \\
\leq & C \sum_{|k-j| \leq 4} 2^{\left(3 / p^{\prime}\right) k}\left\|\widehat{u_{k}}\right\|_{L^{p}}\|v\|_{F \dot{B}_{2 p /(3 p-2), 2}^{-3 / p^{\prime}}} .
\end{aligned}
$$

By (4) in Proposition 3, we know that $L^{2}=F \dot{B}_{2,2}^{0} \mathrm{C}$ $F \dot{B}_{2 p /(3 p-2), 2}^{-3 / p^{\prime}}$. Thus

$$
\left(\sum_{j} 2^{(1-\beta) j 2}\|\widehat{I I}\|_{L^{2}}^{2}\right)^{1 / 2} \leq C\|u\|_{F \dot{B}_{p, 2}^{1-\beta+3 / p^{\prime}}}\|v\|_{L^{2}}
$$

Finally we derive the estimate of the last part as

$$
\begin{aligned}
& \left(\sum_{j} 2^{(1-\beta) j 2}\left\|\widehat{I I I_{j}}\right\|_{L^{2}}^{2}\right)^{1 / 2} \\
& \leq\left\|\sum_{k \geq j-3}\right\| 2^{(1-\beta) j} \varphi_{j}(\xi) \\
& \quad \times\left[\widehat{u_{k}} * \sum_{|l-k| \leq 1} \widehat{v_{l}}\right]\|\|_{L_{\xi}^{2}} \|_{l^{2}(j)} \\
& \leq \sum_{k}\left(\int_{R^{3}} \sum_{j \leq k+3} 2^{(1-\beta) 2 j}\left|\varphi_{j}(\xi)\right|^{2}\right. \\
& \left.\left.\times \sup _{\xi}\left(\sum_{j} \varphi_{j}(\xi)^{2}\right)^{1 / 2} \sum_{|l-k| \leq 1} \widehat{v}_{l}\right]^{2} d \xi\right)^{1 / 2}
\end{aligned}
$$




$$
\begin{aligned}
& \times \sum_{k} 2^{(1-\beta)(k+3)}\left\|\widehat{u}_{k} * \sum_{|l-k| \leq 1} \widehat{v}_{l}\right\|_{L_{\xi}^{2}} \\
\leq & C \sum_{k} 2^{\beta k}\left\|\widehat{u_{k}}\right\|_{L^{2}} \sum_{|l-k| \leq 1} 2^{(1-2 \beta)(k-l)} 2^{(1-2 \beta) l}\left\|\widehat{v}_{l}\right\|_{L^{1}} \\
\leq & C\|u\|_{\dot{H}^{\beta}}\|v\|_{F \dot{B}_{1,2}^{1-2 \beta}} .
\end{aligned}
$$

Now we can begin our proof of Theorem 10. The method is based on the work from Gallagher-Iftimie-Planchon [27].

Proof of Theorem 10. Let $\epsilon>0$ be any constant small enough such that $\epsilon \leq C_{0} \mu$, where $C_{0}$ is the constant in Theorem 5 and $\mu$ is the viscosity coefficient in (1). For $k \in N^{+}$, define

$$
\mathscr{A}_{k}:=\left\{\xi \in R^{3}:|\xi| \leq k \text { and }\left|\widehat{u_{0}}(\xi)\right| \leq k\right\} .
$$

Obviously $\mathscr{F}^{-1}\left(\chi_{\mathscr{A}_{k}} \widehat{u_{0}}\right)$ converges to $u_{0}$ in $F \dot{B}_{p, q}^{1-2 \beta+3 / p^{\prime}}$. So there exists some $k \in N^{+}$such that

$$
\left\|u_{0}-\mathscr{F}^{-1}\left(\chi_{\mathscr{A}_{k}} \widehat{u_{0}}\right)\right\|_{F \dot{B}_{p, q}^{1-2 \beta+3 / p^{\prime}}}<\frac{\epsilon}{2} .
$$

Set

$$
\begin{gathered}
u_{0, k}=\mathscr{F}^{-1}\left(\chi_{\mathscr{A}_{k}} \widehat{u_{0}}\right), \\
w_{0, k}=u_{0}-\mathscr{F}^{-1}\left(\chi_{\mathscr{A}_{k}} \widehat{u_{0}}\right) .
\end{gathered}
$$

Thus $u_{0, k} \in F \dot{B}_{p, q}^{1-2 \beta+3 / p^{\prime}} \cap L^{2}$ and we have shown that $\left\|w_{0, k}\right\|_{F \dot{B}_{p, q}^{1-2 \beta+3 / p^{\prime}}}<\epsilon / 2$. Now we consider the following equations:

$$
\begin{gathered}
w_{t}+\mu(-\Delta)^{\beta} w+(w \cdot \nabla) w \\
+\nabla \pi=0, \quad(t, x) \in R^{+} \times R^{3} \\
\nabla \cdot w=0, \quad(t, x) \in R^{+} \times R^{3} ; \\
w(0, x)=w_{0, k}(x), \quad x \in R^{3}
\end{gathered}
$$

Since $\epsilon / 2 \leq C_{0} \mu / 2 \leq C_{0} \mu$, by Theorem 5 , there exists a unique global solution $w_{k}$ of (72) such that

$$
w_{k} \in \mathscr{C}\left([0, \infty) ; F \dot{B}_{p, q}^{1-2 \beta+3 / p^{\prime}}\right) \cap \mathscr{L}^{1}\left([0, \infty) ; F \dot{B}_{p, q}^{1+3 / p^{\prime}}\right) .
$$

\section{Moreover,}

$$
\begin{aligned}
& \left\|w_{k}\right\|_{\mathscr{L}^{\infty}\left([0, \infty) ; F \dot{B}_{p, q}^{1-2 \beta+3 / p^{\prime}}\right)}+\mu\left\|w_{k}\right\|_{\mathscr{L}^{1}\left([0, \infty) ; F \dot{B}_{p, q}^{1+3 / p^{\prime}}\right)} \\
& \quad \leq C\left\|w_{0, k}\right\|_{F \dot{B}_{p, q}^{1-2 \beta+3 / p^{\prime}}} .
\end{aligned}
$$

An easy computation gives $w_{k} \in C\left([0, \infty) ; F \dot{B}_{p, q}^{1-2 \beta+3 / p^{\prime}}\right)$ and for all $t \in[0, \infty)$, we have

$$
\begin{aligned}
& \left\|w_{k}(t)\right\|_{F \dot{B}_{p, q}^{1-2 \beta+3 / p^{\prime}}}+\mu\left\|w_{k}\right\|_{\mathscr{L}^{1}\left([0, t) ; F \dot{B}_{p, q}^{1+3 / p^{\prime}}\right)} \\
& \quad \leq C\left\|w_{0, k}\right\|_{F \dot{B}_{p, q}^{1-2 \beta+3 / p^{\prime}}} .
\end{aligned}
$$

Now put $u_{k}=u-w_{k}$. Then $u_{k} \in C\left([0, \infty) ; F \dot{B}_{p, q}^{1-2 \beta+3 / p^{\prime}}\right)$ and it satisfies

$$
\begin{gathered}
\partial_{t} u_{k}+\mu(-\Delta)^{\beta} u_{k}+\left(u_{k} \cdot \nabla\right) u_{k}+\left(u_{k} \cdot \nabla\right) w_{k} \\
+\left(w_{k} \cdot \nabla\right) u_{k}+\nabla \pi-\nabla \pi_{k}=0 \\
\nabla \cdot u_{k}=0 \\
u_{k}(0, x)=u_{0, k}(x),
\end{gathered}
$$

where $\pi$ and $\pi_{k}$ are the correspond pressures to the solutions $u$ and $w_{k}$, respectively. Taking the $L^{2}$ inner product with $u_{k}$, we have

$$
\begin{aligned}
& \frac{1}{2} \frac{d}{d t}\left\|u_{k}\right\|_{L^{2}}^{2}+\mu\left\|(-\Delta)^{\beta / 2} u_{k}\right\|_{L^{2}}^{2} \\
& \quad \leq\left|\left\langle\left(u_{k} \cdot \nabla\right) w_{k}+\left(w_{k} \cdot \nabla\right) u_{k}, u_{k}\right\rangle\right|
\end{aligned}
$$

To estimate $\left|\left\langle\left(u_{k} \cdot \nabla\right) w_{k}, u_{k}\right\rangle\right|$, we have

$$
\begin{aligned}
\left|\left\langle\left(u_{k} \cdot \nabla\right) w_{k}, u_{k}\right\rangle\right|= & \left|\left\langle\nabla \cdot\left(u_{k} \otimes w_{k}\right), u_{k}\right\rangle\right| \\
\leq & \left\|(-\Delta)^{1 / 2-\beta / 2}\left(u_{k} \otimes w_{k}\right)\right\|_{L^{2}} \\
& \times\left\|(-\Delta)^{\beta / 2} u_{k}\right\|_{L^{2}} .
\end{aligned}
$$

By Lemma 17, we have

$$
\begin{aligned}
& \left|\left\langle\left(u_{k} \cdot \nabla\right) w_{k}, u_{k}\right\rangle\right| \\
& \leq C\left\|w_{k}\right\|_{F \dot{B}_{p, q}^{1-\beta+3 / p^{\prime}}}\left\|u_{k}\right\|_{L^{2}}\left\|u_{k}\right\|_{\dot{H}^{\beta}} \\
& \quad+C\left\|w_{k}\right\|_{F \dot{B}_{p, q}^{1-2 \beta+3 / p^{\prime}}}\left\|u_{k}\right\|_{\dot{H}^{\beta}}^{2} .
\end{aligned}
$$

By (75), we have $\left\|w_{k}\right\|_{F \dot{B}_{p, q}^{1-2 \beta+3 / p^{\prime}}} \leq C \epsilon / 2$. We further assume $\epsilon$ small enough such that $C^{2} \epsilon \leq \mu / 4$. Using $a b \leq a^{2} / 2+b^{2} / 2$, we have

$$
\begin{aligned}
\left|\left\langle\left(u_{k} \cdot \nabla\right) w_{k}, u_{k}\right\rangle\right| \leq & \frac{2 C^{2}}{\mu}\left\|w_{k}\right\|_{F \dot{B}_{p, q}^{1-\beta+3 / p^{\prime}}}^{2}\left\|u_{k}\right\|_{L^{2}}^{2} \\
& +\frac{\mu}{4}\left\|u_{k}\right\|_{\dot{H}^{\beta}}^{2} .
\end{aligned}
$$

Thus we conclude that

$$
\frac{d}{d t}\left\|u_{k}\right\|_{L^{2}}^{2}+\mu\left\|u_{k}\right\|_{\dot{H}^{\beta}}^{2} \leq \frac{8 C^{2}}{\mu}\left\|w_{k}\right\|_{F \dot{B}_{p, q}^{1-\beta+3 / p^{\prime}}}^{2}\left\|u_{k}\right\|_{L^{2}}^{2} .
$$


Gronwall lemma gives

$$
\begin{aligned}
\left\|u_{k}\right\|_{L^{2}}^{2}+\mu \int_{0}^{t}\left\|u_{k}\right\|_{\dot{H}^{\beta}}^{2} & \leq\left\|u_{0, k}\right\|_{L^{2}}^{2} \\
& \times \exp \left\{\frac{8 C^{2}}{\mu} \int_{0}^{t}\left\|w_{k}\right\|_{F \dot{B}_{p, q}^{1-\beta+3 / p^{\prime}}}^{2}\right\} .
\end{aligned}
$$

Since $q \leq 2$, by Minkowski's inequality, we get

$$
\begin{gathered}
\int_{0}^{t}\left\|w_{k}\right\|_{F \dot{B}_{p, q}^{1-\beta+3 / p^{\prime}}}^{2} \\
\leq\left(\sum_{j \in Z} 2^{j\left(1-\beta+3 / p^{\prime}\right) q}\right. \\
\left.\quad \times\left(\int_{0}^{t}\left\|\varphi_{j} \widehat{w_{k}}\right\|_{L^{p}}^{2}\right)^{q / 2}\right)^{2 / q} \\
\leq\left(\sum_{j \in Z} 2^{j\left(1-2 \beta+3 / p^{\prime}\right) q / 2}\left\|\varphi_{j} \widehat{w}_{k}\right\|_{L^{\infty}\left([0, t) ; L^{p}\right)}^{q / 2}\right. \\
\left.\quad \times 2^{j\left(1+3 / p^{\prime}\right) q / 2}\left\|\varphi_{j} \widehat{w_{k}}\right\|_{L^{1}\left([0, t) ; L^{p}\right)}^{q / 2}\right)^{2 / q} \\
\leq\left\|w_{k}\right\|_{\mathscr{L}^{\infty}\left([0, t) ; F \dot{B}_{p, q}^{1-2 \beta+3 / p^{\prime}}\right)}\left\|w_{k}\right\|_{\mathscr{L}^{1}\left([0, t) ; F \dot{B}_{p, q}^{1+3 / p^{\prime}}\right)} .
\end{gathered}
$$

Thus, combining with (74) we can obtain

$$
\begin{aligned}
& \left\|u_{k}\right\|_{L^{2}}^{2}+\mu \int_{0}^{t}\left\|u_{k}\right\|_{\dot{H}^{\beta}}^{2} \\
& \quad \leq\left\|u_{0, k}\right\|_{L^{2}}^{2} \exp \left\{\frac{8 C^{4}}{\mu^{2}}\left\|w_{0, k}\right\|_{F \dot{B}_{p, q}^{1-2 \beta+3 / p^{\prime}}}^{2}\right\} .
\end{aligned}
$$

Using Lemma 16 with $s=\beta$ and (75), we know that $u_{k} \in$ $L^{4 \beta /(5-4 \beta)}\left([0,+\infty) ; F \dot{B}_{p, q}^{1-2 \beta+3 / p^{\prime}}\right)$ and

$$
\begin{aligned}
\int_{0}^{\infty} & \left\|u_{k}\right\|_{F \dot{B}_{p, q}^{1-2 \beta+3 / p^{\prime}}}^{4 \beta /(5-4 \beta)} \\
\leq & C^{4 \beta /(5-4 \beta)} \mu^{-1}\left\|u_{0, k}\right\|_{L^{2}}^{4 \beta /(5-4 \beta)} \\
& \times \exp \left\{\frac{16 C^{4} \beta}{\mu^{2}(5-4 \beta)}\left\|w_{0, k}\right\|_{F \dot{B}_{p, q}^{1-2 \beta+3 / p^{\prime}}}^{2}\right\} .
\end{aligned}
$$

So by continuity, there is a time $t_{0}$ such that $\left\|u_{k}\left(t_{0}\right)\right\|_{F \dot{B}_{p, q}^{1-2 \beta+3 / p^{\prime}}} \leq \epsilon / 2$. Then we have

$$
\begin{aligned}
\left\|u\left(t_{0}\right)\right\|_{F \dot{B}_{p, q}^{1-2 \beta+3 / p^{\prime}}} \leq & \left\|u_{k}\left(t_{0}\right)\right\|_{F \dot{B}_{p, q}^{1-2 \beta+3 / p^{\prime}}} \\
& +\left\|w_{k}\left(t_{0}\right)\right\|_{F \dot{B}_{p, q}^{1-2 \beta+3 / p^{\prime}}} \\
\leq & \frac{\epsilon}{2}+\frac{\epsilon}{2} \leq \epsilon .
\end{aligned}
$$

Finally, we consider the fractional Navier-Stokes equations starting at $t=t_{0}$; by Theorem 5 and using a method as estimating (75) we conclude that

$$
\begin{gathered}
\|u(t)\|_{F \dot{B}_{p, q}^{1-2 \beta+3 / p^{\prime}}}+\mu\|u\|_{\mathscr{L}^{1}\left(\left[t_{0}, t\right) ; F \dot{B}_{p, q}^{1+3 / p^{\prime}}\right)} \\
\leq C\left\|u\left(t_{0}\right)\right\|_{F \dot{B}_{p, q}^{1-2 \beta+3 / p^{\prime}}} \leq C \epsilon .
\end{gathered}
$$

for all $t>t_{0}$. Thus we finish our proof.

\section{Conflict of Interests}

The authors declare that there is no conflict of interests regarding the publication of this paper.

\section{Acknowledgment}

The research was supported by the NNSF of China under Grants no. 11271330 and no. 11201103.

\section{References}

[1] J. Leray, "Sur le mouvement d'un liquide visqueux emplissant l'espace," Acta Mathematica, vol. 63, no. 1, pp. 193-248, 1934 (French).

[2] H. Fujita and T. Kato, "On the Navier-Stokes initial value problem. I," Archive for Rational Mechanics and Analysis, vol. 16, pp. 269-315, 1964.

[3] T. Kato, "Strong $L^{p}$-solutions of the Navier-Stokes equation in $R^{m}$, with applications to weak solutions," Mathematische Zeitschrift, vol. 187, no. 4, pp. 471-480, 1984.

[4] M. Cannone, Wavelets, Paraproducts and Navier-Stokes: With a Preface by Yves Meyer, Diderot, Paris, France, 1995, (French).

[5] H. Koch and D. Tataru, "Well-posedness for the Navier-Stokes equations," Advances in Mathematics, vol. 157, no. 1, pp. 22-35, 2001.

[6] J. Bourgain and N. Pavlović, "Ill-posedness of the NavierStokes equations in a critical space in 3D," Journal of Functional Analysis, vol. 255, no. 9, pp. 2233-2247, 2008.

[7] A. Cheskidov and R. Shvydkoy, "Ill-posedness of the basic equations of fluid dynamics in Besov spaces," Proceedings of the American Mathematical Society, vol. 138, no. 3, pp. 1059-1067, 2010.

[8] J.-L. Lions, Quelques Méthodes de Résolution des Problèmes aux Limites non Linéaires, Dunod, Paris, France, 1969.

[9] J. Wu, "Generalized MHD equations," Journal of Differential Equations, vol. 195, no. 2, pp. 284-312, 2003.

[10] J. Wu, "The generalized incompressible Navier-Stokes equations in Besov spaces," Dynamics of Partial Differential Equations, vol. 1, no. 4, pp. 381-400, 2004.

[11] J. Wu, "Lower bounds for an integral involving fractional Laplacians and the generalized Navier-Stokes equations in Besov spaces," Communications in Mathematical Physics, vol. 263, no. 3, pp. 803-831, 2006.

[12] J. Xiao, "Homothetic variant of fractional Sobolev space with application to Navier-Stokes system," Dynamics of Partial Differential Equations, vol. 4, no. 3, pp. 227-245, 2007.

[13] P. Li and Z. Zhai, "Well-posedness and regularity of generalized Navier-Stokes equations in some critical Q-spaces," Journal of Functional Analysis, vol. 259, no. 10, pp. 2457-2519, 2010. 
[14] P. Li and Z. Zhai, "Generalized Navier-Stokes equations with initial data in local Q-type spaces," Journal of Mathematical Analysis and Applications, vol. 369, no. 2, pp. 595-609, 2010.

[15] Z. Zhai, "Well-posedness for fractional Navier-Stokes equations in critical spaces close to $\dot{B}_{\infty, \infty}^{-2 \beta-1}\left(R^{n}\right)$," Dynamics of Partial Differential Equations, vol. 7, pp. 25-44, 2010.

[16] X. Yu and Z. Zhai, "Well-posedness for fractional Navier-Stokes equations in the largest critical spaces $\dot{B}_{\infty, \infty}^{-(2 \beta-1)}\left(R^{n}\right)$," Mathematical Methods in the Applied Sciences, vol. 35, no. 6, pp. 676683, 2012.

[17] A. Cheskidov and R. Shvydkoy, "Ill-posedness for subcritical hyperdissipative Navier-Stokes equations in the largest critical spaces," Journal of Mathematical Physics, vol. 53, Article ID 115620, 2012.

[18] C. Deng and X. Yao, "Well-posedness and ill-posedness for the 3D generalized Navier-Stokes equations in $\dot{F}_{(3 / \alpha-1)}^{-\alpha, r}$," Discrete and Continuous Dynamical Systems. Series A, vol. 34, no. 2, pp. 437-459, 2014.

[19] M. Cannone and G. Karch, "Smooth or singular solutions to the Navier-Stokes system?" Journal of Differential Equations, vol. 197, no. 2, pp. 247-274, 2004.

[20] A. Biswas and D. Swanson, "Gevrey regularity of solutions to the 3-D Navier-Stokes equations with weighted $l \backslash s b p$ initial data," Indiana University Mathematics Journal, vol. 56, no. 3, pp. 1157-1188, 2007.

[21] P. Konieczny and T. Yoneda, "On dispersive effect of the Coriolis force for the stationary Navier-Stokes equations," Journal of Differential Equations, vol. 250, no. 10, pp. 3859-3873, 2011.

[22] D. Fang, B. Han, and M. Hieber, "Global existence results for the Navier-Stokes equations in the rotational framework," http://arxiv.org/abs/1205.1561.

[23] Z. Lei and F. Lin, "Global mild solutions of Navier-Stokes equations," Communications on Pure and Applied Mathematics, vol. 64, no. 9, pp. 1297-1304, 2011.

[24] M. Cannone and G. Wu, "Global well-posedness for NavierStokes equations in critical Fourier-Herz spaces," Nonlinear Analysis. Theory, Methods \& Applications, vol. 75, no. 9, pp. 3754-3760, 2012.

[25] Z. Zhang and Z. Yin, "Global well-posedness for the generalized Navier-Stokes sysytem," http://arxiv.org/abs/1306.3735.

[26] J. Benameur, "Long time decay to the Lei-Lin solution of 3D Navier-Stokes equations," http://arxiv.org/abs/1312.2136.

[27] I. Gallagher, D. Iftimie, and F. Planchon, "Non-explosion en temps grand et stabilité de solutions globales des équations de Navier-Stokes," Comptes Rendus Mathematique, vol. 334, no. 4, pp. 289-292, 2002. 


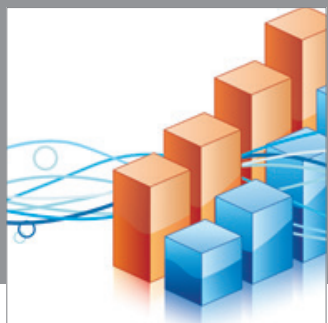

Advances in

Operations Research

mansans

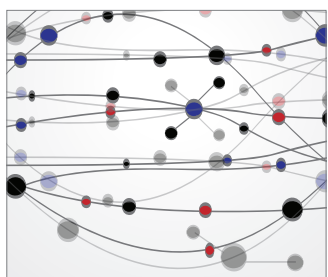

The Scientific World Journal
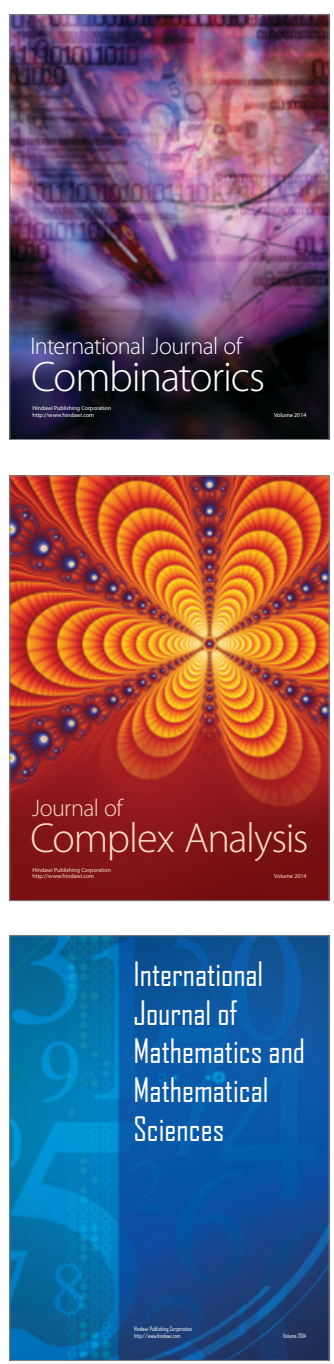
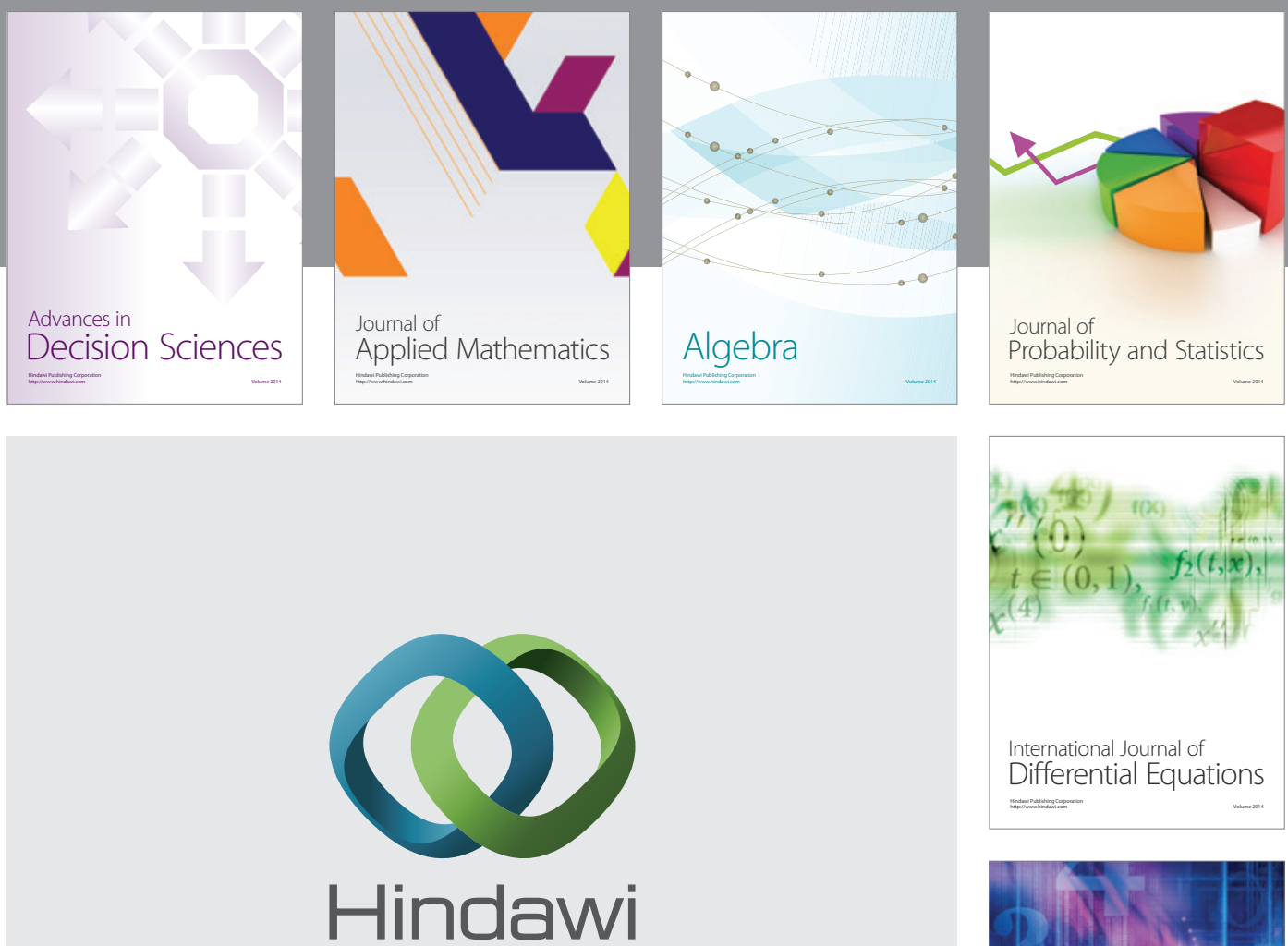

Submit your manuscripts at http://www.hindawi.com
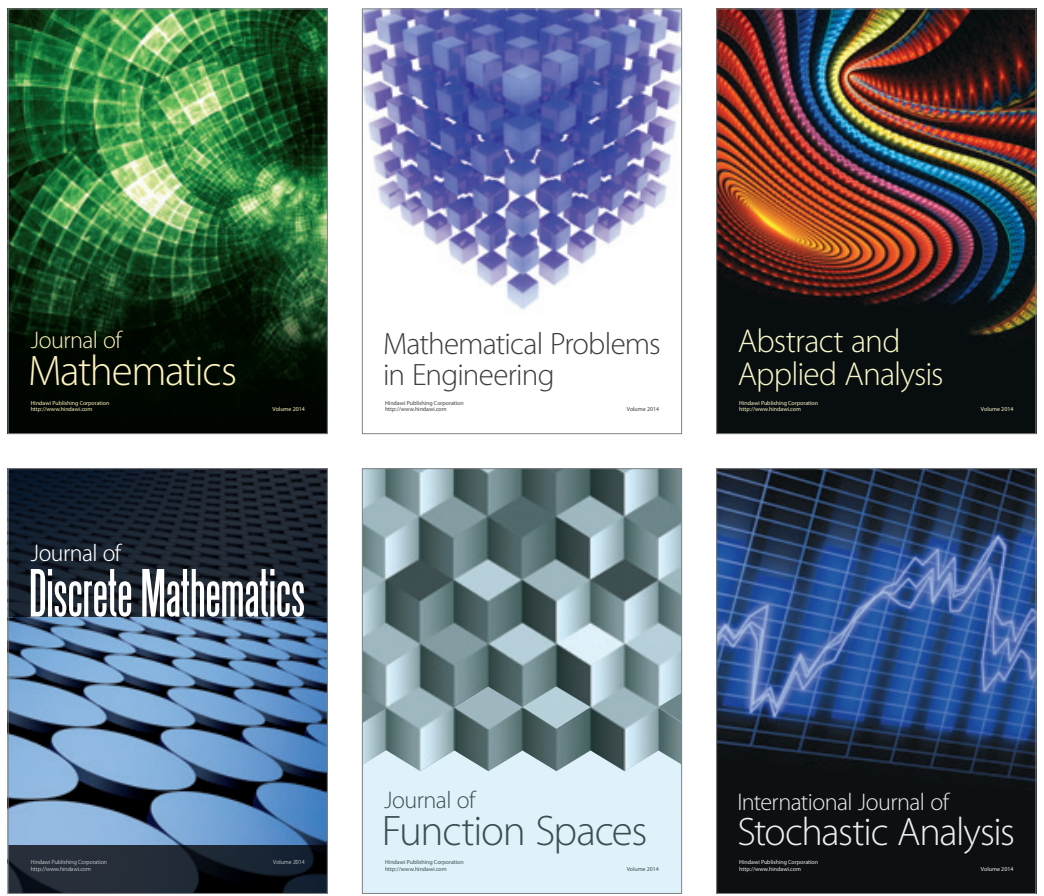

Journal of

Function Spaces

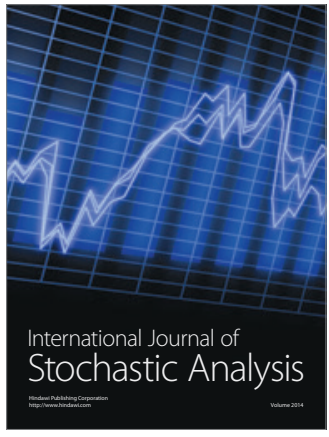

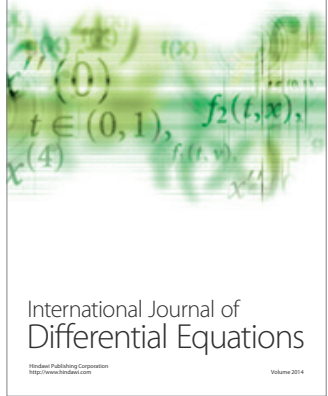
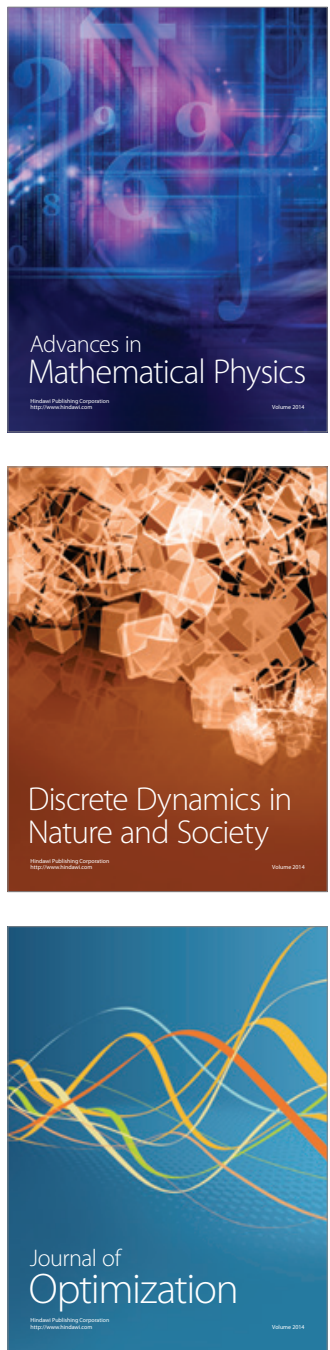